\title{
DE PLANTIS HISPANIAE NOTULAE SYSTEMATICAE, CHOROLOGICAE ET ECOLOGICAE, II
}

\author{
S. RIVAS-MARTínEZ *
}

\section{RESUMEN}

Se publican algunos datos sistemáticos, corológicos y ecológicos sobre la Flora Española. Además se proponen las siguientes nuevas combinaciones: Phlomis purpurea L. subsp. almeriensis ( $\mathrm{Pau}$ ) Losa \& Rivas Goday; Phlomis purpurea L. subsp. caballeroi (Pau) Rivas-Martínez; Phlomis italica L. subsp. antiatlantica (Peltier) RivasMartínez; Hormathoprylla lapeyrousiana (Jordan) Küpfer subsp. angustifolia (Willk.) Rivas-Martínez; Avenula sulcata (Gay ex Delastre) Dumort. subsp. albinervis (Boiss.) Rivas-Martínez.

\section{ABSTRACT}

Some systematic, chorologic and ecological data of Spanish Flora are included in this papel. Also we proposed the following new combinations: Phlomis purpurea L. subsp. almeriensis (Pau) Losa \& Rivas Goday; Phlomis purpurea L. subsp. caballeroi (Pau) Rivas-Martínez; Phlomis italica L. subsp. antiatlantica (Peltier) Rivas-Martínez; Hormathophylla lapeyrousiana (Jordan) Küpfer subsp. angustifolia (Willk.) Rivas-Martínez; Avenula sulcata (Gay ex Delastre) Dumort. subsp. albinervis (Boiss.) Rivas-Martínez.

\section{INTRODUCCIÓN}

Se continúa en este artículo las notas sistemáticas, corológicas y ecológicas sobre Flora Española que se iniciaron con el mismo título hace poco tiempo: Candollea, 31 (1): 111-117 (1976).

\section{Euphorbia prostrata Aiton, Hort. Kew 2: 139 (1789)}

Adventicia de origen norteamericano, extendida por toda la región mediterránea occidental, que se halla con frecuencia en la provincia de Madrid. Este pequeño terófito de floración tardía tiene claras preferencias ecológicas

(*) Departamento de Botánica. Facultad de Farmacia. Universidad Complutense. Madrid. 
por los biótopos pisoteados, en particular por los caminos enlosados adyacentes a suelos limo-arenosos.

Algunos inventarios realizados en comunidades donde vive E. prostrata Aiton, nos ha permitido reconocer una asociación bastante extendida al menos por el Centro de España (Euphorbietum chamaesyco-prostratae), en la que dominan los neófitos de origen norteamericano, y que tiene su homóloga en Barcelona en el Eleusino-Euphorbietum prostratae (O. Bolós, 1962).

Un inventario realizado en la Ciudad Puerta de Hierro (Madrid), 6-XI1976, cob. $50 \%$, área 0,5 m cuadrados, que consideramos tipo de la asociación, tenía la siguiente composición florística: 2.3 Euphorbia prostrata Aiton; 2.2 Euphorbia maculata L.; 1.2 Euphorbia chamaesyce subsp. chamaesyce; 1.2 Polygonum aviculare L.; +.2 Poa infirma Humb., Bonpl. \& Kunth; +.2 Cynodon dactylon (L.) Pers.; +Amaranthus deflexus L.; + Anacyclus clavatus (Desf.) Pers.; + Conyza canadensis (L.) Cronq. La asociación (Euphorbietum chamaesyco-prostratae) y sus homólogas europeas de desarrollo estivo-autumnal (Eleusino-Euphorbietum prostratae) pertenecen a la clase Polygono-Poetea annuae (Rivas-Martínez, 1975), en cuyo seno pueden constituir una nueva alianza: Euphorbion prostratae (síntipo: Euphorbietum chamaesyco-prostratae), de areal análogo al del Polycarpion tetraphylli pero distinta fenología y caracterizada por un buen número de neófitos de origen americano: Euphorbia prostrata Aiton, Euphorbia maculata L., Euphorbia chamaesyce L., Euphorbia serpens Kunth, Amaranthus blitoides S. Watson, Amaranthus deflexus L.

A. \& O. DE Bolós (1950) publicaron una excelente clave para separar cuatro especies (E. maculata, E. prostrata, E. chamaesyce, E. serpens) de las seis existentes en las Comarcas Barcelonesas del subgénero Chamaesyce Rafin (incl. E. nutans Lag., E. peplis L.), que adaptamos para las tres especies reconocidas hasta ahora en la provincia de Madrid pertenecientes a dicho subgénero.

1. Entrenudos de las ramas iguales o más largos que las hojas; lámina entera o solo dentada en el ápice y casi tan larga como ancha; cápsula glabra o pelosa, con los pelos patentes dispuestos en

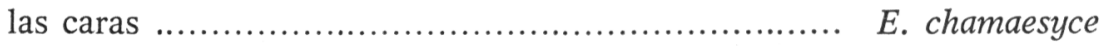

1. Entrenudos de las ramas más cortos que las hojas, lámina de borde aserrado o denticulado y obviamente más larga que ancha; cápsula pelosa

2. Cápsula con pelos largos $(0,5 \mathrm{~mm})$ dispuestos solamente en la quilla, estípulas triangulares E. prostrata

2. Cápsula uniformemente cubierta de pelos cortos (0,1-0,2 mm) adpresos, estípulas laciniado-setáceas E. maculata 
12. Phlomis purpurea L. subsp. almeriensis (Pau) Losa \& Rivas Goday comb. nova

Phlomis purpurea L., endemismo ibero-mauritano, está diversificado en la Península Ibérica al menos en dos subespecies: a) subsp. purpurea, de hojas anchas y dientes del cáliz provistos de un mucrón por lo menos tan largo como el diente (Phlomidetalia purpureae); b) subsp. almeriensis, de hojas estrechas y dientes del cáliz provistos de un mucrón menor que el diente (Anthyllidetalia).

La combinación a nivel de subespecie de la var. almeriensis Pau sigue siendo ilegítima, pese a haber sido utilizada repetidas veces por nosotros (Rivas Goday \& Rivas-Martínez, 1969). Por ello se aprovecha esta ocasión para darla validez. Phlomis purpurea L. subsp. almeriensis (Pau) Losa \& Rivas Goday comb. nova. Bas. Phlomis purpurea L. var. almeriensis Pau, Mem. Mus. Ci. Nat. Barcelona (Bot.) 1(3): 29 (1925) 三Phlomis purpurea L. subsp. almeriensis (Pau) Losa \& Rivas Goday in Rivas Goday \& RivasMartínez, Anal. Inst. Bot. Cavanilles, 25: 168 (1969) nom. illeg.

Phlomis caballeroi Pau, frecuente en Marruecos y en estos momentos con duda en Almería (Rivas Goday \& Rivas-Martínez, 1969), tiene las hojas adultas verdosas por la cara superior, los dientes del cáliz ovado obtusos y el mucrón corto subescondido entre los tricomas. En mi opinión debe considerarse como un extremo de la variación del Phlomis purpurea L., y subordinarse como subespecie. Phlomis purpurea L. subsp. caballeroi (Pau) Rivas-Martínez comb. nova. Bas. Phlomis caballeroi Pau, Bol. Soc. Aragon. Ci. Nat. 17: 132 (1918).

Phlomis italica L., endemismo balear, tiene un taxon vicario (subsp. antiatlantica) en las montañas del Anti-Atlas marroquí, que me parece debe ser subordinado al Ph. italica como subespecie. Phlomis italica L. subsp. antiatlantica (Peltier) Rivas-Martínez stat. nov. Bas. Phlomis antiatlantica Peltier, Candollea, 31: 5 (1976), isotypi G.

Clave para determinar los Phlomis comentados

1. Bracteolas florales lineares de menos de $2 \mathrm{~mm}$ de ancho; dientes del cáliz redondeados más anchos que largos; corola menor de $20 \mathrm{~mm} \ldots 2$

1. Bracteolas florales elípticas de $2-5 \mathrm{~mm}$ de ancho; dientes del cáliz acuminados al menos tan largos como anchos; corola mayor de $20 \mathrm{~mm} \mathrm{...} 3$

2. Cáliz tubuloso, ápice de los mericarpos glabros .. Ph. italica subsp. italica

2. Cáliz ligeramente infundibuliforme, ápice de los mericarpos provistos de pelos estrellados $P h$. italica subsp. antiatlantica 
3. Página superior de las hojas adultas verdoso glabrescente, dientes del cáliz tan largos como anchos, mucrón corto subescondido entre el tomento Ph. purpurea subsp. caballeroi

3. Página superior de las hojas adultas blanquecino pubescente; dientes del cáliz más largos que anchos, mucrón largo visible ................ 4

4. Hojas oval-lanceoladas ligeramente cordadas en la base; mucrón igual o mayor que el diente del cáliz Ph. purpurea subsp purpurea

4. Hojas lanceoladas truncadas en la base; mucrón menor que el diente del cáliz Ph. purpurea subsp. almeriensis

13. Festuca eskia Ramond ex DC. in Lam. \& DC. Fl. Fr. 3:52 (1805)

Especie de origen pirenaico presente en la Cordillera Cantábrica y que hemos herborizado en el macizo de Peña Ubiña. Merino la cita de los Ancares, y A. Segura (1969), gran conocedor de la Flora Española, de la Sierra de Urbión. Según KüPFER (1974) toda la especie es diploide $(2 n=14)$ y llegaría hasta los Cárpatos orientales. En el espolón SE de Peña Ubiña, a $2.300 \mathrm{~m}$, se encontraba abundante en compañía de Luzula pediformis (Chaix) DC., sobre suelos profundos descarbonatados.

En el valle de San Emiliano y en el macizo de Peña Ubiña existe un buen número de táxones del género Festuca. En los cervunales y prados higrófilos de Torrebarrio abunda Festuca rubra L. subsp. multiflora (Hoffm.) Jirasek ex Dostal. En todos los prados de siega, desde San Emiliano hasta Puerto Ventana, es común Festuca pratensis Hudson que a veces vive en compañía de Festuca rubra L. subsp. nevadensis Hackel var. gaetula Maire in St. Yves. En los prados higrófilos más ricos en nitrógeno de los alrededores de San Emiliano es común Festuca arundinacea Schreber subsp. uechtritziana (Wiesb.) Hackel ex Hegi. En los hayedos septentrionales basífilos de Puerto Ventana (Saxifrago hirsutae-Fagetum) merece la pena citar Festuca altissima All. Y, por último, en todos los matorrales de enebros, gayubas y sabinas, es frecuente, por encima de los $1.600 \mathrm{~m}$, Festuca hervieri Patzke, que sustituye altitudinalmente en los suelos calcáreos a Festuca hystrix Boiss., muy común en el piso de los sabinares albares (Juniperetum sabino-thuriferae).

14. Lolium parabolicum Sennen ex Samp., Bol. Soc. Broteriana (ser. 2), $1: 125$ (1922).

Terófito de pequeño porte, hojas cortas arrolladas y espigas contraídas, que abunda en las comunidades psamófilas algo nitrificadas, de las dunas interiores de las playas gallegas. Playa de Corme (La Coruña), Playa de San Ciprián (Lugo). 
15. Avenula sulcata (Gay ex Delastre) Dumort. subsp. albinervis (Boiss.) Rivas-Martínez stat. nov.

Tanto el taxon diploide (Avena sulcata Gay ex Delastre) como el tetraploide (Avena albinervis Boiss.) se hallan en los pastizales silicícolas de la Cordillera Central. El diploide prefiere los cervunales o prados de Nardus stricta L. (Nardetalia) y el tetraploide los lastonares o pastos vivaces de Festuca elegans Boiss. y F. durandoi Claus. (Festucion elegantis). Aunque falta la confirmación cariológica parece que son interfértiles en la naturaleza, donde hemos hallado con frecuencia poblaciones morfológicamente intermedias (Sierras de Gredos, Bejar y Estrela). Por todo ello, creemos que el status más adecuado para ambos táxones es el de subespecie, y así lo proponemos: Avenula sulcata (Gay ex Delastre) Dumort. subsp. albinervis (Boiss.) Rivas-Martínez stat. nov. Bas. Avena albinervis Boiss., Voy. Bot. Esp., 2: 656 (1844) $\equiv$ Avenochloa albinervis (Boiss.) Holub, Act. Hort. Bot. Prag., 1: 82 (1962) 三Avenula albinervis (Boiss.) Laínz, Comunicación INIA (ser. Rec. Nat.), 2: 23 (1974).

16. Oreochloa elegans Sennen, Bull. Soc. Bot. Fr. 74:406 (1927).

$\mathrm{Si}$ admitimos como especie independiente Oreochloa blanka Deyl, Opera Bot. Cechica, 3:244 (1946), taxon diploide de distribución pirenaicocantábrica, el nombre de SENNEN descrito de los Pirineos orientales (Kerguelen, 1975), tendría prioridad sobre el de Deyl. No así en el rango de subespecie, cuyo nombre válido sería el de Oreochloa disticha (Wulfen) Link subsp. blanka (Deyl) Küpfer, Boissiera, 23:53 (1974). KüPFER (1974) sugiere que ambos táxones son diploides, $2 \mathrm{n}=14$, esquizoendémicos, y acreedores del rango subespecífico; subsp. disticha (alpino-carpático), subsp. blanka (pirenaico-cantábrico).

17. Juniperus sabina L., Sp. Pl. 1.039 (1753).

Abundaba en la arista suroccidental de Peña Ubiña (Cordillera Cantábrica), a $2.200 \mathrm{~m}$, en comunidades de enebros y sabinas (Pino-Juniperion sabinae). Un inventario realizado allí (área $40 \mathrm{~m}$ cuadrados, incl. $25 \%$, cob. 95 \%) mostraba: 3.4 Juniperus sabina L., 2.3. Juniperus nana Willd., 2.3 Arctostaphilos uva-ursi (L.) Spreng., +.2 Genista legionensis Pau, +.2 Oreochloa seslerioides (All.) K. Richter subsp. confusa (Coincy) Laínz, +.2 Ranunculus parnassifolius L. subsp. favargeri Küpfer (= Ranunculus aloissiceballii Guinea). 
18. Hormathophylla lapeyrousiana (Jordan) Küpfer subsp. angustifolia (Willk.) Rivas-Martínez stat. nov.

El menor tamaño constante de la var. angustifolia, su mayor pubescencia, y, sobre todo, sus hojas más cortas dispuestas en roseta, permiten separarla de la var. lapeyrousiana. Por demás, su corología y afinidades fitosociológicas diferentes: var. lapeyrousiana (Aphyllanthenion), var. angustifolia (Salvienion lavandulaefoliae), parecen aconsejar en este caso el rango subespecífico que proponemos: Hormathophylla lapeyrousiana (Jordan) Küpfer subsp. angustifolia (Willk.) Rivas-Martínez stat. nov. Bas. Ptilotrichum peyrousianum (Gay) Willk. var. angustifolia Willk. in Willk. \& Lange, Prodr. Fl. Hisp. 3:835 (1880) 三 Hormathophylla lapeyrousiana (Jordan) Küpfer var. angustifolia (Willk.) Küpfer, Boissiera, 23 : 214 (1974).

\section{BIBLIOGRAFIA}

Bolós, A. \& col. Bolós, O. 1950. Vegetación de las Comarcas Barcelonesas. Instituto Español de Estudios Mediterráneos, Barcelona.

Bolós, O. 1962. El paisaje vegetal barcelonés. Universidad de Barcelona, 1-193, Barcelona.

Kergú́len, M. 1975. Les gramineae (Poaceae) de la Flore Française. Essai de mise au point taxonomique et nomenclaturale. Lejeunia, no. 75, 343 pp. Liege.

KüPFER, P. 1974. Recherches sur les liens de parenté entre la flore orophile des Alpes et celle des Pyrenées. Boissiera, 23: 1-322.

Rivas Goday, S. \& Rivas-Martínez, S. 1969. Matorrales y tomillares de la Península Ibérica comprendidos en la clase Ononido-Rosmarinetea Br.-Bl. 1947. Anal. Inst. Bot. Cavanilles, 25: 5-201, Madrid.

Rivas-Martínez, S. 1975. Sobre la nueva clase Polygono-Poetea annuae. Phytocoenologia, 2 (1/2): 123-140, Stuttgart-Lehre.

Segura Zubizarreta, A. 1969. Notas de Flora Soriana (Herbario del Distrito Forestal de Soria). Inst. Forest. Invest. Exper. Comunicación n. ${ }^{\circ} 52,71$ pp. Madrid. 